\title{
MET targeting: perspectives for the radiation oncologist
}

Cyrus Chargari and Eric Deutsch

We read with great interest the Review by Solange Peters and Alex Adjei (MET: a promising anticancer therapeutic target. Nat. Rev. Clin. Oncol. 9, 314-326; 2012) on targeting the MET pathway as an anticancer target. ${ }^{1}$ In this high-quality paper, the authors focus on the current challenges and the most promising drug combinations of conventional chemotherapy and targeted agents. ${ }^{1}$ Using the example of rilotumumab, the authors briefly underscored the preclinical rationale of inhibiting the MET pathway in combination with radiotherapy. ${ }^{2}$ We would like to further highlight how MET is involved in radiation resistance and how targeting the hepatocyte growth factor (HGF)-MET activation loop shows promise for enhancing the antitumour effects of radiotherapy.

Recently, De Bacco et al. ${ }^{3}$ investigated this issue and demonstrated that ionizing radiation could induce the expression and activity of the MET oncogene. After irradiation, MET expression in breast cancer cell lines was increased up to fivefold, and this overexpression increased ligandindependent MET phosphorylation and signal transduction, which protects cells from apoptosis. ${ }^{3}$ Cells that survived irradiation also became increasingly invasive, an effect that was driven through activation of the ataxia telangiectasia mutated (ATM) and nuclear factor $\kappa \mathrm{B}(\mathrm{NF}-\kappa \mathrm{B})$ signalling pathways.

The role of the stroma in this process should be highlighted. The epithelialmesenchymal transition refers to the acquisition of a fibroblast phenotype as well as increased cellular migration and proliferation in response to irradiation. ${ }^{4}$ This programme is partially controlled by HGF, which is produced by irradiated fibroblasts. HGF is involved in ionizingradiation-induced cell invasion and in the frequent metastatic relapses observed in patients after radiotherapy. ${ }^{3}$ These effects are counterbalanced in vivo by treatment with MET inhibitors, which enhance the efficacy of radiotherapy, at least in a xenograft animal model. ${ }^{3}$

The induction of the ATM pathway is consistent with other reports that suggest MET is involved in DNA repair after exposure to radiotherapy. ${ }^{2,5}$ Indeed, MET inhibition has been shown to impair the formation of the RAD51-BRCA2 complex, which is involved in the homologous repair of double-strand DNA breaks. ${ }^{6}$ Additionally, $M E T$ expression correlates with increased hypoxia, which is the most powerful factor of resistance to ionizing-radiation therapy. ${ }^{7}$ Pennacchietti et al. ${ }^{7}$ have shown that hypoxia triggers transcription of the MET oncogene as well as amplifies HGF signalling and synergizes with HGF to induce activation of the downstream MET pathway.

Finally, Peters and Adjei note that preclinical data showing how MET inhibitors overcome resistance to EGFR inhibitors have not been recapitulated in patients. ${ }^{1}$ To date, all available clinical data on radiotherapy combined with tyrosine kinase inhibitors that block EGFR have been disappointing. In this setting, targeting MET could be particularly promising for enhancing the effectiveness of radiotherapy alone or when combined with EGFR inhibitors. In fact, surviving cells become resistant to ionizingradiation therapy through enhanced MET signalling and activation of the downstream antiapoptotic PI3K/Akt pathway. ${ }^{3,8}$ Thus, dual inhibition of EGFR and MET could be promising for enhancing the efficacy of radiotherapy.
We are totally in agreement with Peters and Adjei that the use of biomarkers is important for selecting candidates to receive MET inhibitors. ${ }^{1}$ Tomorrow's rationally designed clinical trials should incorporate biomarkers of tumour response or of metastatic dissemination to optimize therapy with MET inhibitors in combination with radiotherapy.

Gustave Roussy Institute, INSERM 1030, 39 Rue Camille Desmoulins, 94805 Villejuif Cedex, France (C. Chargari, E. Deutsch). Correspondence to: E. Deutsch eric.deutsch@igr.fr

\section{Competing interests}

The authors declare no competing interests.

1. Peters, S. \& Adjei, A. A. MET: a promising anticancer therapeutic target. Nat. Rev. Clin. Oncol. 9, 314-326 (2012).

2. Buchanan, I. M. et al. Radiosensitization of glioma cells by modulation of Met signalling with the hepatocyte growth factor neutralizing antibody, AMG102. J. Cell. Mol. Med. 15, 1999-2006 (2011).

3. De Bacco, F. et al. Induction of MET by ionizing radiation and its role in radioresistance and invasive growth of cancer. J. Natl Cancer Inst. 103, 645-661 (2011).

4. Boccaccio, C. \& Comoglio, P. M. Invasive growth: a MET-driven genetic programme for cancer and stem cells. Nat. Rev. Cancer 6, 637-645 (2006).

5. Welsh, J. W. et al. The c-Met receptor tyrosine kinase inhibitor MP470 radiosensitizes glioblastoma cells. Radiat. Oncol. 4, 69 (2009).

6. Medová, M., Aebersold, D. M. \& Zimmer, Y. MET inhibition in tumor cells by PHA665752 impairs homologous recombination repair of DNA double strand breaks. Int. J. Cancer 130, 728-734 (2012).

7. Pennacchietti, S. et al. Hypoxia promotes invasive growth by transcriptional activation of the met protooncogene. Cancer Cell 3 , 347-361 (2003).

8. Zhan, M. \& Han, Z. C. Phosphatidylinositide 3-kinase/AKT in radiation responses. Histol. Histopathol. 19, 915-923 (2004). 